\section{(6) OPEN ACCESS}

Department of Paediatric Gastroenterology,

The Children's Centre,

The General Infirmary at Leeds, Leeds, UK

\section{Correspondence to}

Dr Veena Zamvar, Department of Paediatric Gastroenterology, The Children's Centre, Paediatric Offices, Room 21 (Block 16) A Floor, Brotherton Wing, The General Infirmary at Leeds, Great George Street, Leeds, West Yorkshire, LS1 3EX, UK; veenazamvar@hotmail.com

Received 4 May 2012 Accepted 18 October 2012 Published Online First 9 November 2012

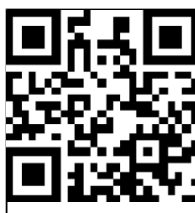

To cite: Zamvar $V$ Lazonby G, Puntis JWL. Arch Dis Child 2013:98: 556-557.

\title{
Recurrent life-threatening sepsis in intestinal failure: transplantation or foster care?
}

\author{
Veena Zamvar, Gill Lazonby, John W L Puntis
}

\begin{abstract}
Parenteral nutrition has transformed the outlook for patients with intestinal failure, but is associated with serious long-term complications, including catheterrelated blood stream infection, liver disease and loss of venous access. Risks can be significantly reduced by strict adherence to management regimens, such as catheter-care protocols, but intestinal transplantation is indicated when complications threaten survival. The responsibility of home parenteral nutrition as an alternative to long-term hospitalisation is welcomed by many parents, but represents a huge burden of care that sometimes proves beyond their capacity. We report two children with recurrent life-threatening central venous catheter infections who were removed from the intestinal transplant list following virtual cessation of infective episodes after going into foster care. These cases raise important issues about the level of family support offered, the role of non-compliance with treatment routines in relation to risk of complications, and how this should be assessed and monitored.
\end{abstract}

\section{INTRODUCTION}

Home parenteral nutrition (HPN) has transformed the management of intestinal failure (IF), improving quality of life for patients. ${ }^{1}$ However, PN not only places a major burden of care on parents, but is associated with serious complications, such as catheter-related blood stream infection (CRBSI), liver disease, loss of venous access and thromboembolism. $^{2}$ Intestinal transplantation (ITx) is indicated when complications threaten survival. We report two children with recurrent life-threatening CRBSI who were removed from the ITx list when foster care placement led to virtual cessation of septic episodes. These cases raise important issues about the level of family support offered for IF patients, the role of non-compliance with treatment routines in relation to risk of complications, and how this should be assessed.

Patient 1 was an 8-month-old girl with short bowel syndrome (SBS) from complicated gastroschisis $(25-30 \mathrm{~cm}$ small bowel, no ileo-caecal valve, distal colon) who was discharged on HPN to willing and technically competent parents. The family was assisted by the hospital nutrition support team (NST) and community nurses in managing complex social, financial and housing issues. Over the next 3 years, she experienced 32 episodes of CRBSI requiring admission for a total of 655 days, intensive care on 3 occasions, and 10 central venous catheter (CVC) changes. After assessment in a supraregional transplant unit, ITx was recommended on the basis of recurrent lifethreatening sepsis. Parents had shown increasing reluctance to respond to signs of sepsis and seek medical attention, and visited the ward infrequently. Our perception was that they struggled to maintain the high standard of care required, and following discussion, they admitted difficulty in coping with the demands of HPN. Social care agencies declined further support until child protection concerns were emphasised; she was subsequently discharged into foster care aged three-and-a-half years, and transplantation deferred pending review of clinical progress. Over the next 5 years, she experienced only one episode of CRBSI, spending around 10 days in hospital each year for assessment and CVC replacement following fracture or occlusion.

Patient 2 was a 3 -month-old infant with SBS secondary to neonatal volvulus $(11 \mathrm{~cm}$ small bowel, intact ileo-caecal valve, colon) was discharged on HPN to her motivated and competent single mother. Over the first 15 months, she had 5 episodes of CRBSI, requiring 68 days in hospital; during the next 15 months she had 16 episodes of CRBSI, 4 admissions to intensive care; 7 CVC changes and 144 inpatient days. Following assessment in the transplant unit, she was also recommended for ITx. The NST and community nurses became increasingly concerned over changes in the mother's behaviour, including delay in seeking medical attention, poor compliance with treatment, substandard CVC care, and inappropriate administration of medications in mistaken attempts to prevent hospital admission. The mother agreed she was struggling to meet the demands of $\mathrm{HPN}$; depression was subsequently diagnosed and treated. Following discussions with the family and social care agencies, the child went into voluntary foster care with grandparents. Over the next 3 years, there were no further episodes of CRBSI, and one CVC change after dislodgement. During this time, she was taken off the transplant list.

Patient 1 has recently undergone ITx because of poor venous access from widespread venous thrombosis, while patient 2 has returned to mother's care, and is doing well with three nights of PN weekly.

\section{DISCUSSION}

Developments in home care have transformed the management of chronic illnesses, but place immense responsibilities on families. While arrangements vary in different countries, in the UK, what support is made available to carers (home care 'packages') is jointly funded by the community medical service (Clinical Commissioning Group) and welfare services (social care). A scoring system is used to ascertain the level of dependency for 
each patient, and this in turn, determines the level of support accorded to carers. For instance, children requiring $24 \mathrm{~h}$ care (eg, tracheostomy) automatically score highly, so that parents are then eligible for respite care and additional support that might include home nursing. By contrast, HPN currently attracts a low score, and parents are deemed to merit little extra help. However, few parents when faced with the alternative of longterm hospitalisation express unwillingness to take on responsibility for HPN.

The inherent life-threatening risks of HPN are reduced by careful training of carers and compliance with care protocols. ${ }^{1}$ Safe and effective delivery of home care is, therefore, dependent on the ability and motivation of carers to follow the prescribed regimens. Parents need to be adept at daily routines of setting up and flushing off PN, enteral tube feeding, administration of medications, responding to infusion pump alarms and managing symptoms, such as diarrhoea, high stoma output and vomiting. The need to work in conjunction with the NST in a common endeavour to keep the child healthy and out of hospital must be acknowledged; this involves regular exchange of information, and compliance with outpatient visits.

In reality, it is difficult to convey to parents the likely impact of home care on family life. A recent review has highlighted three themes from parental experiences of HPN: physical exhaustion due to troublesome and often nocturnal symptoms; psychosocial and emotional stress due to social isolation; feelings of guilt and financial burden due to job loss. ${ }^{3}$ At times, the burden of responsibility, in addition to other family pressures, may result in poor compliance and adverse outcomes, yet carers can appear technically competent when HPN skills are formally reassessed. Non-compliance arises from an inability to cope emotionally with the stresses surrounding the recommended treatment. Parents may find it difficult to admit to feeling under stress from fear of losing the child and also feelings of guilt. ${ }^{4}$ More extreme parental non-compliance may represent a special form of child abuse; ${ }^{4} 5$ neglect is the most prevalent form of maltreatment in children with chronic illnesses; foster care is the most frequent form of intervention. ${ }^{5}$ Our impression is that those parents with complex family and social circumstances (eg, single parent; unemployment; debt; substance abuse) experience the most difficulties. This problem (which might be termed 'carer fatigue') should be anticipated and discussed from the outset, so that carers are able to raise concerns openly if they feel more help is required. Close supervision by the NST, and working in partnership with families, is essential. In our experience, home visits by the nutrition nurse specialists are very valuable in terms of identifying problems and troubleshooting at an early stage, yet this outreach or community role for hospital-based staff is not always acknowledged by hospital management, or remunerated by commissioners.

Welfare and community agencies need to have a better understanding of the burden of care represented by HPN, and be proactive in providing support; this might need to encompass home nursing and respite care. Change in clinical condition, such as recurrent life-threatening infections, must alert professionals to the possibility of carer fatigue and non-compliance, even though this may initially be denied by the carers themselves. Help for parents should come before blame for inadequacy, and clinical teams need to focus on preventing complications that otherwise become indications for small bowel transplantation. Poor compliance with the management of HPN may well predict poor compliance with complex post-transplantation care regimens. Our cases indicate that a change of carer can sometimes circumvent the need for intestinal transplantation, however, providing additional home care support for families may be more appropriate than instituting child protection proceedings with a view to foster care.

Contributors JWLP was responsible for the original idea and final approval of the manuscript. VZ and GL acquired the information. VZ was responsible for the drafting of the manuscript and subsequent revision with help from JWLP.

Funding None.

Competing interests None.

Patient consent Obtained.

Provenance and peer review Not commissioned; externally peer reviewed.

Open Access This is an Open Access article distributed in accordance with the Creative Commons Attribution Non Commercial (CC BY-NC 3.0) license, which permits others to distribute, remix, adapt, build upon this work non-commercially, and license their derivative works on different terms, provided the original work is properly cited and the use is non-commercial. See: http://creativecommons.org/ licenses/by-nc/3.0/

\section{REFERENCES}

1 Puntis JW. The economics of home parenteral nutrition. Nutrition 1998;14:809-12.

2 Buchman AL. Complications of long-term home total parenteral nutrition: their identification, prevention and treatment. Dig Dis Sci 2001;46:1-18.

3 Sexton E, Coad J, Holden C. Review of homecare packages for paediatric HPN patients. Br J Nurs 2005;14:1080-5.

4 Menahem S, Halasz G. Parental non-compliance-a paediatric dilemma. A medical and psychodynamic perspective. Child Care Health Dev 2000;26:61-72.

5 Jaudes PK, Diamond LJ. Neglect of chronically ill children. Am J Dis Child 1986;140:655-8. 